\title{
Avaliação do uso da Classificação Internacional de Doenças para codificar a síndrome da imunodeficiência adquirida
}

\section{Evaluation of the use of the International Classification of Diseases for the codification of AIDS}

\author{
Cássia Maria Buchalla, Ruy Laurenti, Ana Freitas Ribeiro, Décio Rogério Nitrini, Célia Elisa \\ Guarnieri, Elione Lopes Carnaúba e Maria Aparecida C. Costa Neves
}

Departamento de Epidemiologia da Faculdade de Saúde Pública da Universidade de São Paulo. São Paulo, SP - Brasil (C.M.B., R.L.), Serviço de Epidemiologia do Instituto de Infectologia Emílio Ribas. São Paulo, SP - Brasil (A.F.R., D.R.N., C.E.G., E.L.C., M.A.C.C.N.)

\begin{abstract}
Resumo
A Classificação Internacional de Doenças (CID) provê códigos para todas as doenças e permite que se façam comparações internacionais da morbidade e da mortalidade. Por ser ampla e de uso em locais com características bastante diversas, nem sempre é adequada. Existem revisões periódicas da CID para que esta seja atualizada e as novas doenças descritas sejam incluídas. Foi o que aconteceu com a AIDS, incorporada no capítulo de doenças infecciosas e parasitárias da décima revisão da CID. Por esse motivo, foi avaliado o uso dos códigos da CID-10 na codificação da AIDS. Foram utilizadas as fichas de vigilância epidemiológica de todos os casos diagnosticados em um hospital especializado em doenças infecciosas, em 1994. Verificou-se que nem sempre era possível codificar as fichas apenas com a utilização de um único código, proposto pela CID-10, para a AIDS e suas manifestações e complicações.
\end{abstract}

Classificação Internacional de Doenças. Síndrome de imunodeficiência adquirida, classificação.

\begin{abstract}
The International Classification of Diseases (ICD) provides codes for all conditions and permits international comparisons of morbidity and mortality. Because it has to be used all over the world its codes are not always perfectly appropriate. There are revisions from time to time to bring up-to-date the classification. This has happened with AIDS, included in the chapter of infectious diseases in the last revision, ICD-10. The use of ICD-10 AIDS codes is here appraised. All surveillance report forms of one specialized hospital in infectious diseases, notified in 1994, have been codified, but it was found that many diagnoses could not be codified in accordance with a single ICD-10 AIDS code.
\end{abstract}

International Classification of Diseases. Acquired immunodeficiency syndrome, classification.

Correspondência para / Correspondence to: Cássia Maria Buchalla - Centro Colaborador da OMS para Classificação de Doenças em Português. Faculdade de Saúde Pública da Universidade de São Paulo. Av. Dr. Arnaldo, 715 - 01246-904 São Paulo, SP - Brasil. Fax: (55.11) 883-4246. E-mail: cmbuchal@usp.br.

Edição subvencionada pela FAPESP. Processo 95/2290-6

Recebido em 18.12.1995. Reapresentado em 2.4.1996. Aprovado em 23.4.1996. 


\section{INTRODUÇÃO}

O uso internacional de uma classificação de doenças, para codificar diagnósticos e elaborar estatísticas, permite que se façam comparações entre áreas diferentes, inclusive entre vários países. A classificação deve ser abrangente para conter todos os diagnósticos possíveis e satisfazer os locais onde a tecnologia e a atenção médica são bastante desenvolvidas como também locais sem essas características e onde os diagnósticos, algumas vezes, ainda são expressos como sintomas ou sinais.

A Classificação Internacional de Doenças (CID), ainda que permita todas as possibilidades de codificação acima mencionadas, e mesmo no caso do aparecimento de uma doença nova, quando há meios de se encontrar um código para classificá-la, nem sempre ela é totalmente adequada a algumas necessidades. Exemplo pode ser dado em relacão a câncer. A CID classifica as neoplasias de acordo com o comportamento (maligno, benigno, in situ, etc.) e a localização anatômica. Para profissionais da área de oncologia saber se um tumor está na pequena ou na grande curvatura do estômago não é suficiente. A informação adequada seria aquela que registre também o tipo de célula que forma o tumor ou ainda o estadiamento do mesmo. Para contornar essa dificuldade, a classificação permite, com o uso de códigos adicionais de uma adaptação da CID para morfologia dos tumores, a codificação do tipo de célula.

Para incorporar os novos conhecimentos, há revisões periódicas da CID. A última delas, aprovada em 1990, é a Décima Revisão, conhecida como CID$10^{4}$. No período entre revisões, doenças ou possibilidades diagnósticas novas, não contidas na CID, poderiam ser codificadas em um código não específico para essa condição. Ficariam agrupadas com outras doenças ou condições semelhantes. Foi o que ocorreu com a AIDS que foi descrita pela primeira vez durante a vigência da nona revisão, que não apresentava categoria específica para essa síndrome.

Assim, quando, no início dos anos oitenta apareceram os primeiros casos de imunodeficiência adquirida, o código mais adequado foi o 279.1 referente à "deficiência de imunidade celular". Desta forma, as primeiras estatísticas de AIDS continham também casos da síndrome de Di George, da síndrome de Nezelof e da síndrome de Wiskott-Aldrich, todas englobadas na categoria 279.1. Este código é uma subcategoria de 279 que agrupa transtornos envolvendo os mecanismos imunitários, dentro do capítulo de doenças endócrinas, nutricionais, metabólicas e imunitárias da CID- $9^{3}$, totalmente inadequado para conter uma doença infecciosa, causada por um retrovírus, como viria a se saber em 1983.

Em 1986, nos EUA, resolveu-se criar um código para a infecção pelo HIV (vírus da imunodeficiência adquirida) e suas manifestações. Usando categorias vazias existentes no capítulo de doenças infecciosas e parasitárias, os códigos 042, 043 e 044, para os quais não havia diagnóstico previsto, criou-se uma classificação específica para essa síndrome ${ }^{6}$. Desta forma, além da possibilidade de vários códigos identificando cada doença associada à infecção pelo HIV, estes pertencem ao capítulo onde realmente a doença deveria estar classificada. Essa classificação foi adotada nos EUA e, em seguida, pelo Canadá ${ }^{2}$. Os demais países continuaram codificando a AIDS em 279.1.

A CID-10 contempla a doença pelo HIV com um agrupamento de cinco categorias, de B20 a B24. Este agrupamento, elaborado com base na classificação adotada pelos EUA, contém códigos específicos para algumas das doenças oportunistas mais freqüentemente associadas à AIDS. Assim, pode-se, com apenas um código identificar um caso de AIDS com sarcoma de Kaposi, ou AIDS com pneumonia por Pneumocistis carinii. Há também na CID-10 um código para AIDS não especificada (B24). Existem outras categorias para codificar outras circunstâncias relacionadas à AIDS e que não estão contempladas no agrupamento B20-B24 como "estado de infecção assintomática pelo HIV (Z21)" e "contato com e exposição ao HIV (Z20.6)", ou ainda "evidência laboratorial do HIV (R75)". Portanto é uma classificação que contempla várias possibilidades conseqüentes à infecção pelo HIV.

O ideal para codificar uma doença com vários tipos de manifestações e complicações, como é o caso da AIDS, é que cada código possibilite a identificação específica destas manifestações/complicações. As categorias e subcategorias existentes para a AIDS no agrupamento B20-B24 permitem isso apenas para algumas complicações e não para outras. Nesse último caso a identificação, se necessária, terá que ser feita com a utilização de códigos múltiplos.

O objetivo do presente trabalho é verificar o quanto a codificação de casos de AIDS pela CID10 permite identificar as várias complicações com a utilização de apenas um código ou com o uso de códigos múltiplos.

\section{MATERIAL E MÉTODO}

O instrumento utilizado no estudo foram as fichas de vigilância epidemiológica (FV) de AIDS, de um hospital especializado em doenças infecciosas, do ano de 1994. Essas fichas contêm os dados necessários para se conhe- 
cer as manifestações da AIDS, usadas para definir caso da doença, segundo os dois critérios em uso no Brasil: critério CDC modificado e critério de Caracas ${ }^{5}$. Tem-se, assim, de forma ágil e prática, informações fundamentais para se conhecer todos os diagnósticos existentes, o que é adequado para a avaliação da classificação proposta uma vez que lista todas as circunstâncias freqüentes nos casos de AIDS.

Foram diagnosticados no hospital estudado, $1.278 \mathrm{ca}-$ sos de AIDS. A partir de suas FV as manifestações clínicas foram codificadas com o uso da CID-10. Utilizaramse as categorias B20-B24, com as respectivas subcategorias conforme se apresenta no capítulo I, do volume I da CID10, e outros códigos quando apenas um daquele agrupamento não era suficiente.

\section{RESULTADOS E COMENTÁRIOS}

Na Tabela 1 estão as doenças mais freqüentes e que foram possíveis de serem identificadas com apenas um código. Pode-se observar que isso ocorre com a candidíase e a tuberculose, ainda que nesse último caso não foi possível identificar se era pulmonar ou disseminada.

Tabela 1 - Doenças mais freqüentemente associadas a AIDS que podem ser identificadas com apenas um código da CID-10.

\begin{tabular}{lcc}
\hline Doenças mais freqüentes & $\%$ & $\begin{array}{c}\text { Códigos da } \\
\text { CID-10 }\end{array}$ \\
\hline Candidíase & 53,8 & B20.4 \\
Tuberculose pulmonar & 16,7 & B20.0 \\
Tuberculose disseminada & 14,6 & B20.0 \\
Sarcoma de Kaposi & 5,2 & B21.0 \\
Herpes zoster & 5,0 & B20.3 \\
\hline
\end{tabular}

$\mathrm{Na}$ Tabela 2 estão algumas manifestações que foram freqüentes e que, para serem identificadas, necessitam o uso de dois códigos: um para identificar a AIDS e outro, fora do agrupamento B20-B24, para identificar a manifestação.

Tabela 2 - Diagnósticos relacionados a AIDS codificados pela CID-10 com o uso de duplo código.

\begin{tabular}{lrl}
\hline Sintomas e sinais & $\%$ & $\begin{array}{c}\text { Códigos da } \\
\text { CID-10 }\end{array}$ \\
\hline Astenia & 56,8 & B23.8+R53 \\
Caquexia & 56,3 & B23.8+R64 \\
Febre & 38,3 & B23.8+R50.9 \\
"Transtornos do Sistema & & \\
Nervoso" & 32,3 & B23.8+G98 \\
Diarréia & 25,4 & B20.9+A09 \\
Anemia & 18,8 & B23.2+D64.9 \\
Dermatite & 6,2 & B23.8+L30.9 \\
\hline
\end{tabular}

As doenças mais freqüentemente associadas à AIDS foram identificadas com um único código.
Assim, candidíase (B20.4) esteve presente em 53,6\% dos casos e a pneumonia por Pneumocystis carinii B20.6 -, em 29\%.

Um dos maiores problemas atuais nos países em desenvolvimento é a tuberculose. Ela está associada à AIDS com muita freqüência e sua localização é um critério de diagnóstico. Assim, a última definição de caso de AIDS do $\mathrm{CDC}^{1}$ considera a tuberculose pulmonar, em indivíduos HIV positivos, como doença indicativa de AIDS. No Brasil, segundo o critério de pontuação da OPAS/Caracas, necessitase de especificação da tuberculose para que seja considerado caso de AIDS. Desta forma, a ocorrência de tuberculose disseminada, extrapulmonar ou pulmonar não cavitária, especificadas dessa forma, em indivíduos com exame positivo para o HIV, atribui 10 pontos e define um caso de AIDS. Quando a ocorrência for de tuberculose pulmonar, cavitária ou não especificada, o critério de pontuação determina 5 pontos de um total de 10 necessários para que se considere caso de AIDS ${ }^{5}$.

No entanto, a classificação não permite que se identifique se a tuberculose é pulmonar ou extra-pulmonar utilizando apenas um código (B20.0).

Outras ocorrências freqüentes em pacientes infectados pelo HIV também não puderam ser identificadas apenas com o uso de um código da CID-10, como a diarréia que ocorreu em 25,4\% dos casos atendidos no hospital estudado. Para se obter essa informação dois códigos teriam que ser usados: B24 e A09.

Alguns diagnósticos mais freqüentes, como transtorno do sistema nervoso, não podem ser codificados dentro das categorias para AIDS, sendo necessário também dois códigos B24 e G98.

No Brasil ocorrem com bastante freqüência sintomas e sinais, tanto que o critério de Caracas considera esses como meio de definir caso de AIDS para fins de vigilância. Febre, tosse, astenia e caquexia são bastante comuns em pessoas infectadas e podem definir um caso de AIDS. Todos necessitam de mais de um código para identificar sintoma/sinal e infecção pelo HIV.

Ainda que a mais recente revisão da CID apresente um agrupamento para a doença pelo HIV, contendo 5 categorias (B20 a B24) e várias subcategorias, mesmo assim não satisfaz completamente os usuários que tenham como objetivo identificar as manifestações da doença com apenas um código. $\mathrm{O}$ uso de código duplo faz-se necessário em grande parte das situações. Por outro lado, é preciso lembrar que com freqüência o paciente com AIDS apresenta mais de uma complicação e, nesses casos, será sempre necessário o uso de códigos múltiplos. 


\section{REFERÊNCIAS BIBLIOGRÁFICAS}

1. CENTERS FOR DISEASE CONTROL. 1993 Revised classification system for HIV infection and expanded surveillance case definition for AIDS among adolescents and adults. MMWR, 41: 1- 19, 1992.

2. JOHNSON, R. J.et al. Using death certificates to estimate the completeness of AIDS case reporting in Ontário in 19851987. Can. Med. Assoc. J., 141: 537-40, 1989.

3. ORGANIZAÇÃO MUNDIAL DA SAÚDE. Manual $d a$ classificação internacional de doenças, lesões e causas de óbito; $9^{\circ}$ revisão, 1975. São Paulo, Centro Brasileiro para a Classificação de Doenças, 1978.
4. ORGANIZAÇÃO MUNDIAL DA SAÚDE. Classificação estatística internacional de doenças e problemas relacionados à saúde: CID-10. São Paulo, EDUSP, 1994.

5. SECRETARIA DO ESTADO DA SAÚDE. Centro de Vigilância Epidemiológica “Alexandre Vranjac”. Manual de vigilância epidemiológica: síndrome da imunodeficiência adquirida: AIDS; normas e instruções. São Paulo, 1995.

6. WORLD HEALTH ORGANIZATION. Collaborating Center for Classification of Disease for North America. International classification of diseases: clinical modification, 9th rev. Washington, D. C. 1986. 